\title{
Photo- and thermally-induced changes in the optical properties of Ge-S-Se amorphous films
}

\author{
V.M. Rubish ${ }^{1}$, E.V. Gera ${ }^{1}$, M.O. Durcot ${ }^{1}$, M.M. Pop ${ }^{2}$, S.O. Kostyukevich ${ }^{3}$, A.A. Kudryavtsev ${ }^{3}$, \\ O.S. Mykulanynets-Meshko', M.Yu. Rigan ${ }^{1}$ \\ ${ }^{I}$ Uzhgorod Scientific-Technological Center of the Institute for Information Recording, NAS of Ukraine \\ 4, Zamkovi Skhody str., 88000 Uzhgorod,Ukraine, e-mail:center.uzh@gmail.com \\ ${ }^{2}$ Uzhgorod National University, 3, Narodna sq., 88000, Uzhgorod, Ukraine \\ ${ }^{3}$ V. Lashkaryov Institute of Semiconductor Physics, NAS of Ukraine, 41, prospect Nauky, 03028 Kyiv, Ukraine
}

\begin{abstract}
The optical transmissions spectra of amorphous Ge-S-Se films of chemical compositions $\left(\mathrm{GeS}_{2}\right)_{50}\left(\mathrm{GeSe}_{2}\right)_{50}$ and $\left(\mathrm{GeS}_{3}\right)_{50}\left(\mathrm{GeSe}_{3}\right)_{50}$, prepared by thermal evaporation, have been measured over the whole 400 to $800 \mathrm{~nm}$ spectral range. It has been ascertained that annealing of the films leads to the absorption edge shift into the short-wave spectral region. The values of pseudo-gap width $E_{g}$ and film refraction index $n$ have been determined. Changes in optical properties of films are caused by structural transformations taking place in them under laser illumination and annealing.
\end{abstract}

Keywords: chalcogenide amorphous films, transmission spectra, optical properties, photo-structural transformations.

Manuscript received 15.07.13; revised version received 07.09.13; accepted for publication 23.10.13; published online 16.12.13.

\section{Introduction}

Chalcogenide non-crystalline vitreous and amorphous materials attract continuously growing interest caused by wide possibilities of practical applications and as a unique object for scientific investigations. Due to a wide range of photo-induced phenomena, these materials are current and potential candidates for using in optoelectronics and photonics (inorganic photoresists, optical sensors, waveguides, recording media, circuits, gratings and diffraction elements for various applications) [1-8].

From this viewpoint, deeper studied are amorphous films of binary and ternary arsenic chalcogenides that are characterized by lowering the pseudo-gap (photodarkening of films) under light irradiation [1, 3, 5$10]$, which is assumed to originate from photo-induced structural transformations that can be subsequently reversed by annealing near the glass transition temperature.

In recent decades, intensively studied are photoinduced effects in amorphous films of germanium chalcogenides [11-18]. First of all, in view of practical applications, these materials are attractive if accounting the fact that they do not contain the poisonous arsenic. Second, in these films under illumination one can observe both effects of photodarkening and photobleaching (growth of the pseudogap and optical transparency). Moreover, while germanium sulfide films are characterized only by photobleaching [13-15], the films $\mathrm{Ge}_{x} \mathrm{Se}_{100-x}$ demonstrate both effects: photodarkening in Ge-dificient $(x<20)$ and photobleaching in Ge-rich $(x>20)$ samples [11, 12, 16-18].

In this paper, we report the results of investigating the influence of laser irradiation and annealing on transmission spectra and optical parameters of $\mathrm{GeS}_{2}$ $\mathrm{GeSe}_{2}$ and $\mathrm{GeS}_{3}-\mathrm{GeSe}_{3}$ films with the germanium content 25 and 33.3 at.\%, respectively. Our choice of these sections was caused by the following reasons. In [15] we showed that, at the same conditions of illumination, changes in optical parameters of $\mathrm{GeS}_{2}$ films are essentially higher than those in $\mathrm{Ge}_{2} \mathrm{~S}_{3}$ films. For instance, the change in the refraction index $n$ of $\mathrm{GeS}_{2}$ film is practically 3 -fold higher than that in $\mathrm{Ge}_{2} \mathrm{~S}_{3}$ film. 
In the system Ge-Se, the highest changes in optical characteristics (shift of the absorption edge $E_{g}$ and refraction index $n$ ) were observed by us in the films $\mathrm{GeSe}_{2}$ and $\mathrm{GeSe}_{3}$ [17]. Thereof, it is for the films of the above sections one should expect a high level of photoinduced changes in optical parameters.

\section{Experimental}

The bulk glasses $\mathrm{Ce}_{33.3} \mathrm{~S}_{33.85} \mathrm{Se}_{33.85}$ (or $\left(\mathrm{GeS}_{2}\right)_{50}\left(\mathrm{GeSe}_{2}\right)_{50}$ ) and $\mathrm{Ge}_{25} \mathrm{~S}_{37.5} \mathrm{Se}_{37.5}$ (or $\left(\mathrm{GeS}_{3}\right)_{50}\left(\mathrm{GeSe}_{3}\right)_{50}$ ) were prepared using direct synthesis from the corresponding elements in evacuated silica ampoules. The mass of every charge was 20 gram. In the process of synthesis, we used the step-like increase of temperature up to the maximum one $(1250 \mathrm{~K})$. At the temperatures 700,950 and $1250 \mathrm{~K}$, the ampoules were kept for 8,6 and 4 hours, respectively. In what followed, temperature was lowered down to the temperature of melt homogenization $(1100 \ldots 1150 \mathrm{~K})$. The homogenization time was 48 hours. The melts were periodically stirred. After synthesis the ampoules were air-quenched.

Thin films (thickness $\sim 1 \mu \mathrm{m}$ ) were obtained by vacuum evaporation of glasses of corresponding compositions from quasi-closed effusion cells onto nonheated glass substrates. A uniform thickness of layers was provided by planetary rotation of substrates.

Light exposure of films was made using defocused radiation of a semiconductor laser $(\lambda=530 \mathrm{~nm}$, $P=100 \mathrm{~mW}$ ). Annealing of the films was performed in argon atmosphere for 1 and $2 \mathrm{~h}$ at the temperature $423 \mathrm{~K}$.

Optical transmission spectra of the films were measured at $T=300 \mathrm{~K}$ within the range 400 to $800 \mathrm{~nm}$ by using the method [19] with a monochromator МДР-3. The spectral resolution was no worse than $10^{-3} \mathrm{eV}$.

\section{Results and discussion}

Fig. 1 and 2 (curves 1) show transmission spectra for the as-prepared films $\mathrm{Ge}_{25} \mathrm{~S}_{37,5} \mathrm{Se}_{37,5}$ and $\mathrm{Ge}_{33.3} \mathrm{~S}_{33.35} \mathrm{Se}_{33.35}$. It is obvious that with growing the germanium content in the composition of films, the absorption edge is shifted into short-wave spectral range testifying to increase of the pseudo-forbidden gap width $\left(E_{g}\right)$.

The $E_{g}$ value can be determined from the Tauc relation [13]:

$$
\alpha(h v)=\frac{B\left(h v-E_{g}\right)^{2}}{h v},
$$

which is valid within the range of high energies, when the absorption coefficient has values $\alpha(h v) \geq 10^{-4} \mathrm{~cm}^{-1}$. In (1), $h v$ is the photon energy, $B$ - constant that depends on film material and characterizes the slope of the Tauc absorption edge. The parameter $B$ is an interesting parameter, since it can be taken as a measure of disorder. For example, for $\mathrm{GeS}_{2}, \mathrm{GeSe}_{2}, \mathrm{Ge}_{2} \mathrm{Se}_{3}$ and $\mathrm{GeSe}_{3}$ films the values of parameter $B^{1 / 2}$ is 549 [14], 913 [12], 552 [12], and $827 \mathrm{~cm}^{-1 / 2} \mathrm{eV}^{-1 / 2}$ [12], respectively.

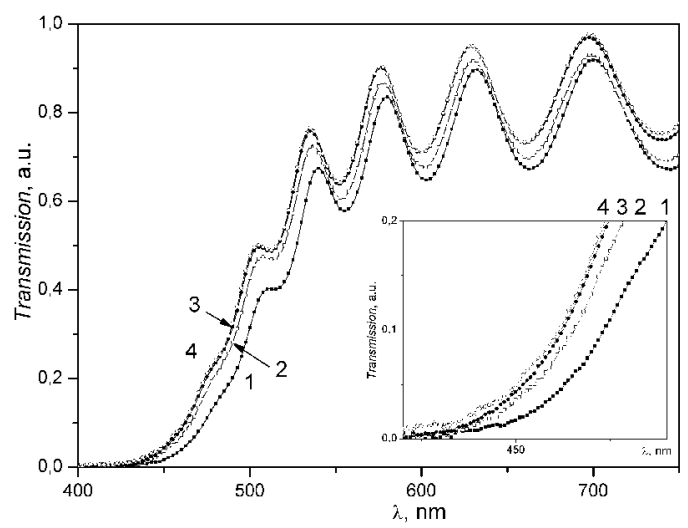

Fig. 1. Dependences of transmission spectra inherent to $\mathrm{Ge}_{25} \mathrm{~S}_{37,5} \mathrm{Se}_{37,75}$ films on the exposure time: $1-0 ; 2-1 ; 3-10$; 4-20 min.

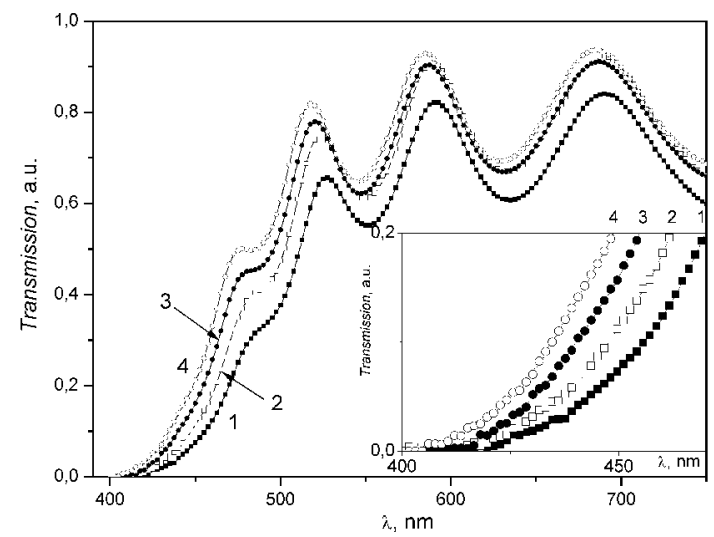

Fig. 2. Dependences of transmission spectra inherent to $\mathrm{Ge}_{33,3} \mathrm{~S}_{33,85} \mathrm{Se}_{33,85}$ films on the exposure time: $1-0 ; 2-1$; 3-10; 4-20 min.

The $E_{g}$ values for $\mathrm{Ge}_{25} \mathrm{~S}_{37.5} \mathrm{Se}_{37.5}$ and $\mathrm{Ge}_{33.3} \mathrm{~S}_{33.35} \mathrm{Se}_{33.35}$ films were determined by extrapolation of the dependences $[\alpha(h v) \cdot h v]^{1 / 2} \sim f(h v)$ down to $\alpha(h v)=0$ (Fig. 3) and are 2.276 and $2.301 \mathrm{eV}$, respectively. For the films $\mathrm{GeS}_{2}, \mathrm{GeSe}_{2}, \mathrm{GeSe}_{3}$, the pseudo-forbidden gap values obtained by us earlier [15, 17] are equal to 2.289 [15], 1.938 [17] and $1.958 \mathrm{eV}$ [17], respectively. These $E_{g}$ values differ to some extent from the pseudo-gap values for the films of the same compositions but obtained by other authors. The determined in $[12,13] E_{g}$ values for the films $\mathrm{GeS}_{2}$, $\mathrm{GeSe}_{2}$ and $\mathrm{GeSe}_{3}$ are equal to 2.53 [13], 2.073 [12] and $2.044 \mathrm{eV}$ [12], respectively. In our opinion, this difference between $E_{g}$ values is caused by technological factors. The refraction index of films was determined using the dependence [20]:

$n=\left[N+\left(N^{2}-s^{2}\right)^{1 / 2}\right]^{1 / 2}$,

where

$N=2 s \frac{T_{\max }-T_{\min }}{T_{\max } \cdot T_{\min }}+\frac{s^{2}+1}{2}$. 

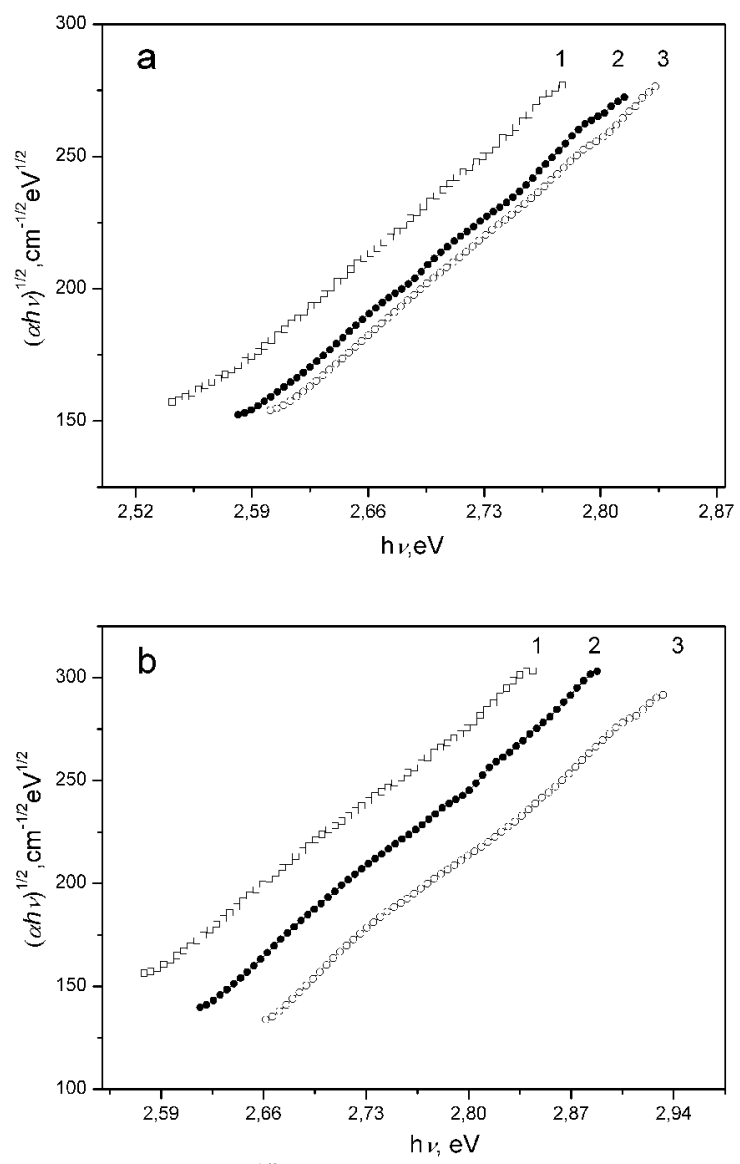

Fig. 3. Plot of $(\alpha h v)^{1 / 2}$ versus $h v$ for the films $\mathrm{Ge}_{25} \mathrm{~S}_{37,5} \mathrm{Se}_{37,75}$ (a) and $\mathrm{Ce}_{33,3} \mathrm{~S}_{33,85} \mathrm{Se}_{33,85}$ (b) non-exposed (1) and exposed (2, 3) for $1(2)$ and $10 \mathrm{~min}(3)$.

In these expressions, $s$ is the refraction index of substrate; $T_{\max }$ and $T_{\min }$ are interferential maxima and minima in transmission spectra within the range of wavelengths where the dispersion of the refraction index is absent. The $n$ values for the films $\mathrm{Ge}_{25} \mathrm{~S}_{37,5} \mathrm{Se}_{37,5}$ and $\mathrm{Ge}_{33,3} \mathrm{~S}_{33,35} \mathrm{Se}_{33,35}$, determined at the wavelength $\lambda=700 \mathrm{~nm}$, are equal to 2.222 and 2.403 , respectively.

Light exposure of films results in a shift of transmission spectra to the short-wave range (Figs 1 and 2 , curves 2 to 4 ). It means that one observes photobleaching of the films. It is indicative of the growth of the pseudo-gap width. After illumination, the refraction index is lowered. The dependences of $E_{g}$ and $n$ values for the films $\mathrm{Ge}_{25} \mathrm{~S}_{37,5} \mathrm{Se}_{37,5}$ and $\mathrm{Ge}_{33,3} \mathrm{~S}_{33,35} \mathrm{Se}_{33,35}$ on the exposure time are depicted in Fig. 4. It is seen that the maximum changes in $E_{g}$ and $n$ values take place at low illumination times. The rate of photo-induced changes in optical parameters is reduced with increasing the irradiation time. In this case, the greater shift of the absorption edge at the transmission level 0.2 (Figs 1 and 2) as well as greater changes of $E_{g}$ and $n$ take place in the film with the Ge content 33.3 at. $\%$ (Fig. 4) under the same exposure conditions.
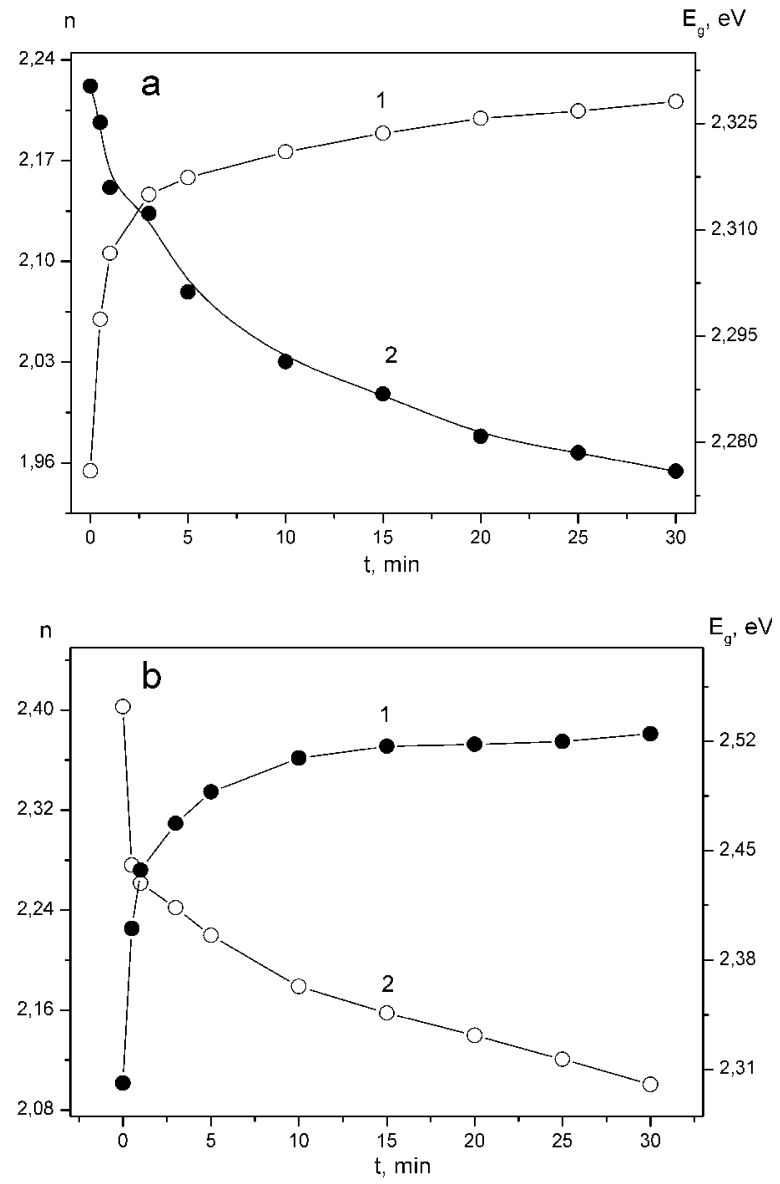

Fig. 4. Dependences of $E_{g}(1)$ and $n(2)$ on the exposure time for $\mathrm{Ge}_{25} \mathrm{~S}_{37,5} \mathrm{Se}_{37,75}$ (a) and $\mathrm{Ge}_{33,3} \mathrm{~S}_{33,85} \mathrm{Se}_{33,85}$ (b) films.

Changes in the absorption edge position (i. e., $E_{g}$ values) and refraction index are caused by structural transformations taking place under laser illumination.

Amorphous films of germanium chalcogenides (Ge-S, Ge-Se, Ge-S-Se, Ge-Sb-S systems) as well as respective glasses possess a nano-heterogeneous structure $[6,12,18,21-29]$. The basic elements of structural network in glasses and films with the germanium content above $25 \%$ are tetrahedrons $\mathrm{GeS}_{n} \mathrm{Se}_{4-n} \quad(n=0-4)$. However, the matrix of germanium chalcogenide glasses and films contains a considerable amount of structural fragments with homopolar bonds Ge-Ge, S-S and Se-Se . In these cases, germanium chalcogenide films contain a higher concentration of homopolar bonds [12, 18, 23]. When illuminating the films, there take place break and reswitch of Ge-Ge, S-S and Se-Se bonds and formation of the heteropolar ones Ge-S(Se) [12, 15, 16, 23]. Photostructural transformations can be also promoted by such structural defects as over-coordinated and undercoordinated atoms of germanium and chalcogen. These processes result in the increase of ordering in the local structure of films. 


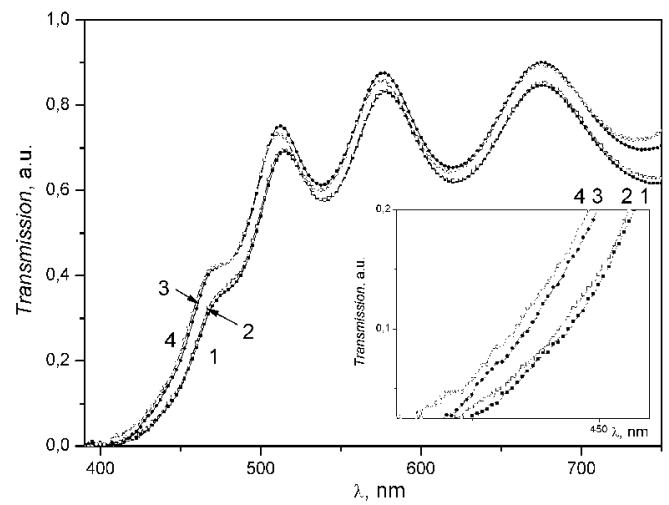

Fig. 5. Transmission spectra of $\mathrm{Ge}_{33,3} \mathrm{~S}_{33,85} \mathrm{Se}_{33,85}$ film annealed for $1 \mathrm{~h}$ at the temperature $423 \mathrm{~K}$ versus the exposure time: $1-0 ; 2-1 ; 3-10 ; 4-20 \mathrm{~min}$.
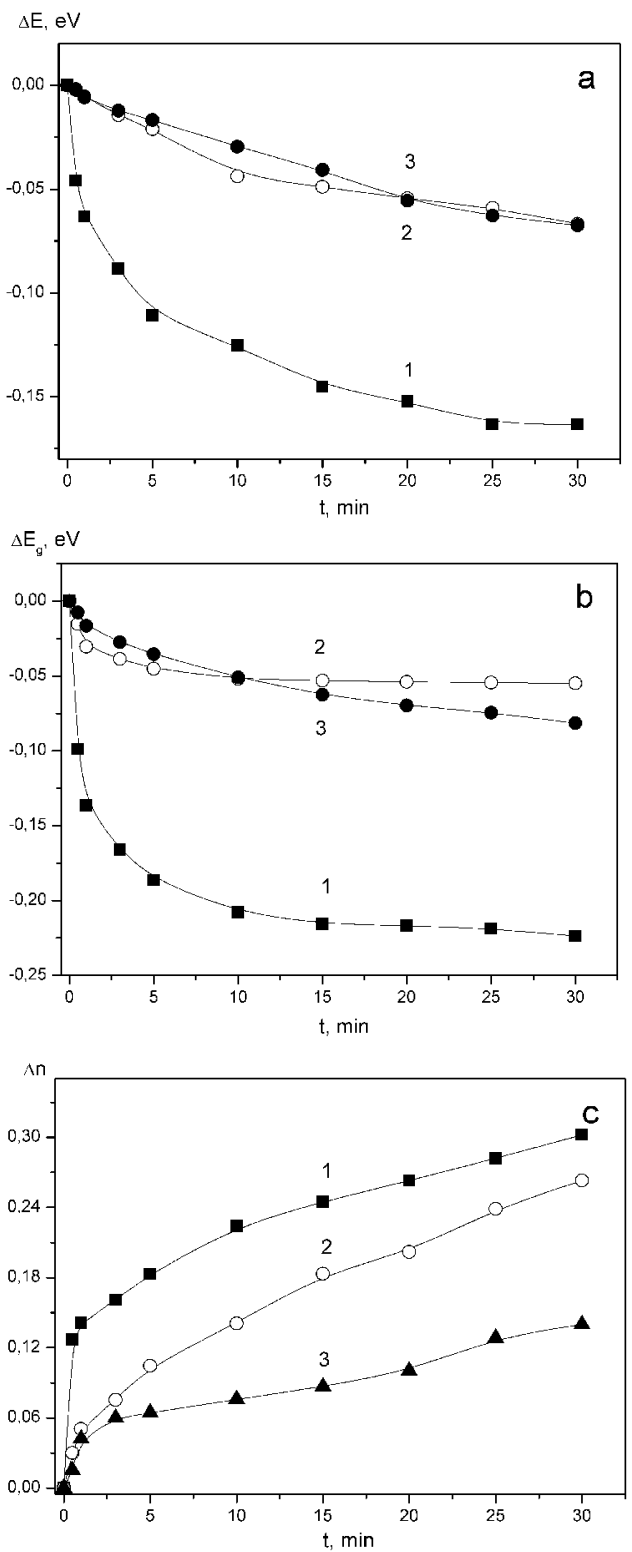

Fig. 6. Shift of the absorption edge at the transmission level 0.2 (a), changes $E_{g}(\mathrm{~b})$ and $n$ (c) versus the exposure time for asprepared (1) and annealed $(2,3)$ at the temperature $423 \mathrm{~K}$ for 1 (2) and $2 \mathrm{~h}(3)$.
With the aim to ascertain the influence of temperature on photo-induced changes in optical characteristic, we investigated transmission spectra of the films $\mathrm{Ge}_{25} \mathrm{~S}_{37.5} \mathrm{Se}_{37.5}$ and $\mathrm{Ge}_{33.3} \mathrm{~S}_{33.35} \mathrm{Se}_{33.35}$ annealed for 1 and $2 \mathrm{~h}$ at the temperature $423 \mathrm{~K}$. It should be noted that this temperature is much lower than the glasstransition temperature for these films.

Thermal annealing of films results in a shift of transmission spectra to the short-wave range. It means that one observes photobleaching of the films. In this case, $E_{g}$ value grows, while $n$ is decreased. So, for the $\mathrm{Ge}_{33.3} \mathrm{~S}_{33.35} \mathrm{Se}_{33.35}$ film annealed for 1 and $2 \mathrm{~h}, E_{g}$ is equal to 2.397 and $2.410 \mathrm{eV}$, respectively. The refraction index values in the same conditions are, respectively, equal to 2.320 and 2.185. In the film $\mathrm{Ge}_{25} \mathrm{~S}_{37.5} \mathrm{Se}_{37.5}$, the degree of $E_{g}$ and $n$ changes is considerably lower.

Thermostimulated changes of Ge-S-Se film optical characteristics are caused by structural transformations. Like to the case of laser illumination, annealing of the films results in break and re-switching the homopolar bonds Ge-Ge, S-S (Se-Se) and formation of structural units with heteropolar bonds $\mathrm{GeS}(\mathrm{Se})_{4 / 2}$.

In this case, thermal polymerization of molecular fragments with homopolar bonds (for example, $\mathrm{Ge}_{2} \mathrm{Se}_{6}$, $\mathrm{Ge}_{2} \mathrm{~S}_{6}, \mathrm{Se}_{2}, \mathrm{~S}_{2}$ ) into the structural network of $\mathrm{GeS}_{n} \mathrm{Se}_{n-4}$ types can be realized both via the defectless mechanism and with creation of structural charged defects (for example, $\left.\mathrm{Ge}_{3}^{-}, \mathrm{S}_{3}{ }^{+}, \mathrm{S}_{1}^{-}[30]\right)$.

Fig. 5 (curves 2 to 4 ) shows transmission spectra for $\mathrm{Ge}_{33.3} \mathrm{~S}_{33.35} \mathrm{Se}_{33.35}$ films illuminated after annealing. It can be seen that the shift of absorption edge $(\Delta E)$ under laser illumination (to the short-wave range) is less in them than that in the as-prepared ones (Fig. 6a). The pseudo-forbidden gap width (Fig. 6b) and refraction index $\Delta n$ changes (Fig. 6c) become considerably lower, too. It means that the level of photo-structural transformations in the annealed films is lower than that in in the as-prepared ones. The lower level of changes in optical parameters in the annealed Ge-S-Se films is conditioned by a rather less number of structural fragments with homopolar bonds in their matrix after annealing, which are able to polymerize under laser irradiation.

\section{Conclusions}

Laser illumination $(\lambda=530 \mathrm{~nm})$ of amorphous films in the system Ge-S-Se leads to the shift in transmission spectra to the short-wave side of the spectrum (photobleaching the films takes place). In this case, the pseudo-forbidden gap width $E_{g}$ grows, while the refraction index value $n$ is lowered. The highest changes in $E_{g}$ and $n$ were observed in the film $\left(\mathrm{GeS}_{2}\right)_{50}\left(\mathrm{GeSe}_{2}\right)_{50}$. These changes of film optical characteristics are caused by photo-structural transformations that are related with the decreasing content of structural fragments possessing homopolar bonds in their matrix. Annealed films show lower level and rate of photo-structural changes in optical parameters. 
References

1. M. Frumar, Z. Cernosek, J. Jedelsky et. al., Photoinduced changes of strucrure and properties of amorphous binary and ternary chalcogenides // $J$. Optoelectron. Adv. Mater. 3 (2), p. 177-188 (2001).

2. V.V. Petrov, A.A. Kryuchin, S.M. Shanoilo, S.A. Kostyukevich, V.G. Kravets, A.S. Lapchuk, Optical Disk: History, State, Perspectives. Naukova dumka, Kyiv, 2003 (in Russian).

3. J. Teteris, M. Reinfelde, Application of amorphous chalcohenide semiconductor thin films in optical recording technologies // J. Optoelectron. Adv. Mater. 5 (5), p. 1335-1360 (2003).

4. V.V. Petrov, A.A. Kryuchin, S.A. Kostyukevich, V.M. Rubish, Inorganic Photolitography. Institute of Metal Physics, NAS of Ukraine, Kyiv, 2007 (in Ukrainian).

5. E.F. Venger, A.V. Melnichuk, A.V. Stronsky, Photostimulated Process in Chalcogenide Vitreous Semiconductors and Their Practical Application. Akademperiodika Publ., Kyiv, 2007 (in Russian).

6. V.V. Petrov, A.A. Kryuchin, V.M. Rubish, Materials for Perspective Optoelectronic Devices. Naukova dunka-Verlag, Kiev, 2012 (in Russian).

7. K. Shimakawa, A.Kolobov, S.R. Elliot, Photoinduced effects and metastability in amorphous semiconductors and insulators // $A d v$. Phys., 44 (6), p. $475-588$ (1995).

8. B.J. Eggleton, B.L. Davies, K. Richardson, Chalcogenide photonics // Nature photonics, 5, p. 141-148 (2011).

9. D. I. Semak, V.M.Rizak, I.M. Rizak, Photothermostructural Transformations of Chalcogenides. Zakarpattya Publ., Uzhgorod, 1999 (in Ukrainian).

10. O.I. Shpotyuk, Yu. N. Shunin, F.V. Pirogov, K.K. Shwarts, V. N. Kornelyuk, Destructionpolymerization Transformations in Chalcogenide Vitreous Semiconductors. Institute of Physics, Latvian AS Publ., Riga, 1991 (in Russian).

11. J. Reyes, E. Marquez, J.B. Ramirez-Malo et al., Optical constants of thermally evaporated amorphous $\mathrm{GeSe}_{3}$ thin films // J. Mater Sci., 30 , p. 4133-4137 (1995).

12. E. Sleeckx, L. Tichy, P. Nagels et al., Thermally and photoinduced irreversible changes in the optical properties of amorphous $\mathrm{Ge}_{x} \mathrm{Se}_{100-x}$ films // J. Non-cryst. Solids 192\&200, p. $723-727$ (1996).

13. K. Petkov, Compositional dependence of the photoinduced phenomena in thin chalcogenide films // J. Optoelectron. Adv. Mater. 4(3), p. 611629 (2002).

14. R. Todorov, Tz. Iliev, K. Petkov, Light-induced changes in the optical properties of thin films of Ge-S-Bi (Te, In) chalcogenides // J. Non-cryst. Solids 326\&327, p. 263-267 (2003).

15. V.M. Maryan, G.T. Horvat, M.M. Pop et al., Photostimulated changes in the optical properties of sulphides germanium and arsenic thin films // Phys.
Chem. Solid State 9 (3), p. 524-528 (2008) (in Ukrainian).

16. V. Lyubin, M. Klebanov, A. Bruner, et. al., Transient photodarkening and photobleaching in glassy $\mathrm{GeSe}_{2}$ films // Optical Mater., 33, p. 949 952 (2011).

17. E.V.Gera, M.M.Pop, M.O.Durcot et al., Photoinduced effects in thin films of Ge-Se systems. .// Mat. $3^{\text {th }}$ Intern. Meeting "Clusters and nanostructured materials (CNM-3)”, Uzhgorod, Ukraine, 2012.

18. R.R. Kumar, A.R. Barik, E.M. Vinod et al., Crossover from photodarkening to photobleaching in a-Ge $\mathrm{Ge}_{100-x}$ thin films // Optics Lett. 38 (10), p. 1682-1684 (2013).

19. I.I. Shpak, I.P. Studenyak, M. Kranjces, Optical absorption edge and structural disorder in electronirradiated $\mathrm{As}_{2} \mathrm{~S}_{3}$ chalcogenide glasses // J. Optoelectron. Adv. Mater. 5(5), p. 1135-1138 (2003).

20. R. Swanepoul, Determination of the thickness and optical constants of amorphous silicon // J. Phys. E: Sci. Instrum. 16, p. 1214-1222 (1983).

21. J. E. Griffits, G.P. Espinosa, J.C. Phillips et al., Raman spectra and thermal laser annealing of $\mathrm{Ge}\left(\mathrm{S}_{x} \mathrm{Se}_{1-x}\right)_{2}$ glasses // Phys. Rev. B 28 (8), p. 44444453 (1983).

22. W. Yong, M. Osamu, I. Koichi et al., A Raman scattering investigation of the strucrure of glassy and liquid $\mathrm{Ge}_{x} \mathrm{Se}_{1-x} / / \mathrm{J}$. Non-cryst. Solids, 232\&234, p. $702-707$ (1998).

23. I.P. Kotsalas, D. Papadimitriou, C. Raptis et al., Raman study of photostructural changes in amorphous $\mathrm{Ge}_{x} \mathrm{Sb}_{0,4-x} \mathrm{~S}_{0,6}$ // J. Non-cryst. Solids. 226, p. 85-91 (1998).

24. P. Boolchand, X. Feng, W.J. Bresser, Rigidity transitions in binary Ge-Se glasses and the intermediate phase // J. Non-Cryst. Solids, 293\&295, p. 85-91 (2001).

25. P.P. Shtets, V.I. Fedelesh, V.M. Kabatsij et al., Structure, dielectric and photoelectric properties of glasses in Ge-Sb-S system // J. Optoelectron. Adv. Mater. 3 (4), p. 937-940 (2001).

26. L. Cai, P. Boolchand, Nanoscale phase separation of $\mathrm{GeS}_{2}$ glass //Phil. Mag. B 82 (15), p. 1649-1657 (2002).

27. P.S. Salmon, Structure of liquidus and glasses in the Ge-Se binary system // J. Non-cryst. Solids, 353, p. 2959-2974 (2007).

28. Jun Zhang, Haiyan Xiao, Jie Zhang. Compositional dependence of refractive index and Raman spectra of $\mathrm{Ge}\left(\mathrm{S}_{x} \mathrm{Se}_{1-x}\right)_{2}$ glasses // J. Optoelectron. Adv. Mater. 13 (7), p. 848-851 (2011).

29. Han Xuecai, Sun Guangying, Liu Yu et al., Structure and vibrational modes of Ge-S-Se glasses: Raman scattering and $\mathrm{AB}$ initio calculations // Chalcogenide Lett. 9 (11), p. 465-474 (2012).

30. O.I. Shpotyuk, R.Ya. Golovchak, A.P. Kovalskiy et al., On the mechanism of radiation-indused optical effects in vitreous $\mathrm{As}_{2} \mathrm{~S}_{3}-\mathrm{GeS}_{2} / / \mathrm{Ukr}$. J. Phys. Opt. 3 (2), p. 134-143 (2002). 